\title{
Desmoid tumor of the stomach
}

A 56-year-old man was referred to our department for evaluation of a gastric mass. His medical history revealed that he had undergone distal gastrectomy for an early gastric carcinoma 18 months previously. Abdominal computed tomography (CT) demonstrated a well-defined, contrastenhancing mass, $3.3 \mathrm{~cm}$ in diameter, with uniform inner structure in the stomach ( Fig. 1). Esophagogastroduodenoscopy (EGD) showed a $3.0-\mathrm{cm}$, submucosal tumor in the greater curvature of the remnant stomach ( $\bullet$ Fig.2a). Endoscopic ultrasound (EUS) of this lesion showed it to be a hypoechoic mass with a sharp boundary in the fourth layer ( $\bullet$ Fig. 2 b).

A clinical diagnosis of gastrointestinal stromal tumor (GIST) of the stomach was made. There was no evidence of further metastatic lesions in other organs, so the patient underwent a completion resection of the remnant stomach.

Gross examination of the resected specimen revealed a round tumor measuring $4.0 \times 4.0 \mathrm{~cm}$ ( $\bullet$ Fig. 3 a). Histologically, the tumor was composed of sheets of spindle-shaped cells with bland nuclear morphology and fine chromatin ( $\bullet$ Fig.3b). Furthermore, immunohistochemistry revealed that the tumor cells were positive for beta-catenin ( Fig.3c). The pathologic diagnosis was therefore desmoid tumor of the stomach and the patient remains alive, with no signs of disease recurrence, 1 year after the operation.

To our knowledge, this is the first report of a patient with a desmoid tumor of the stomach that was treated with gastrectomy. Desmoid tumors, which were first described by John Macfarlane in 1832, are rare, slow growing, benign fibrous neoplasms with no metastatic potential but a strong tendency to invade locally and recur $[1,2]$. The occurrence of desmoid tumors is reported to be associated with female sex, familial adenomatous polyposis (FAP), estrogen therapy, and occasionally with surgical trauma [3].

In our case a man with no family history of FAP but a history of gastrectomy for early
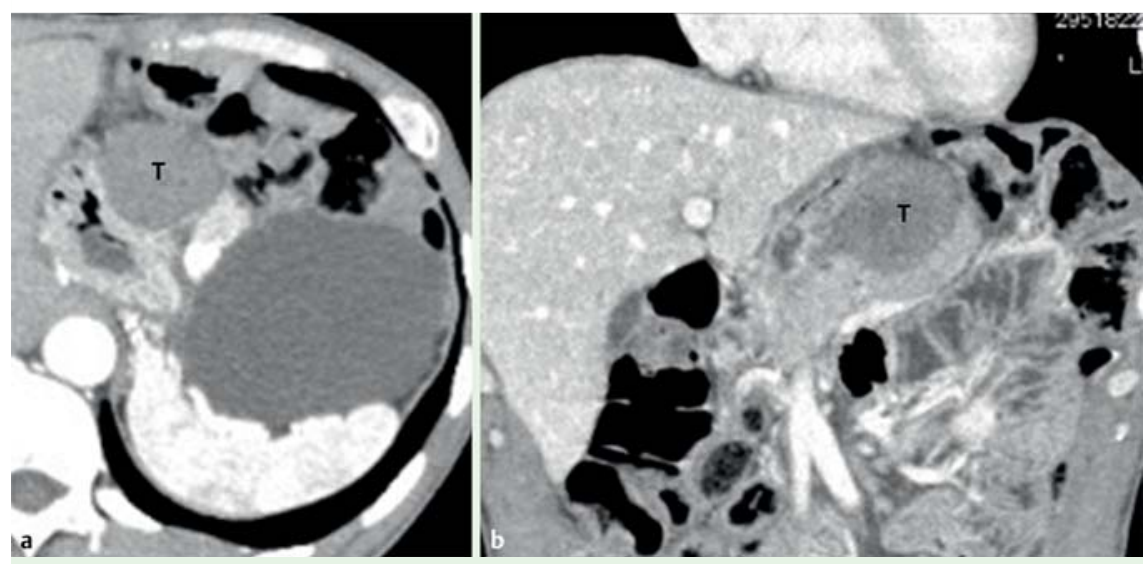

Fig. 1 Abdominal computed tomography $(\mathrm{CT})$ scan in a man who had undergone previous distal gastrectomy showing a $30 \times 33-\mathrm{mm}$, enhancing tumor mass in the stomach $(\mathrm{T})$ in: a horizontal section; b coronal section.
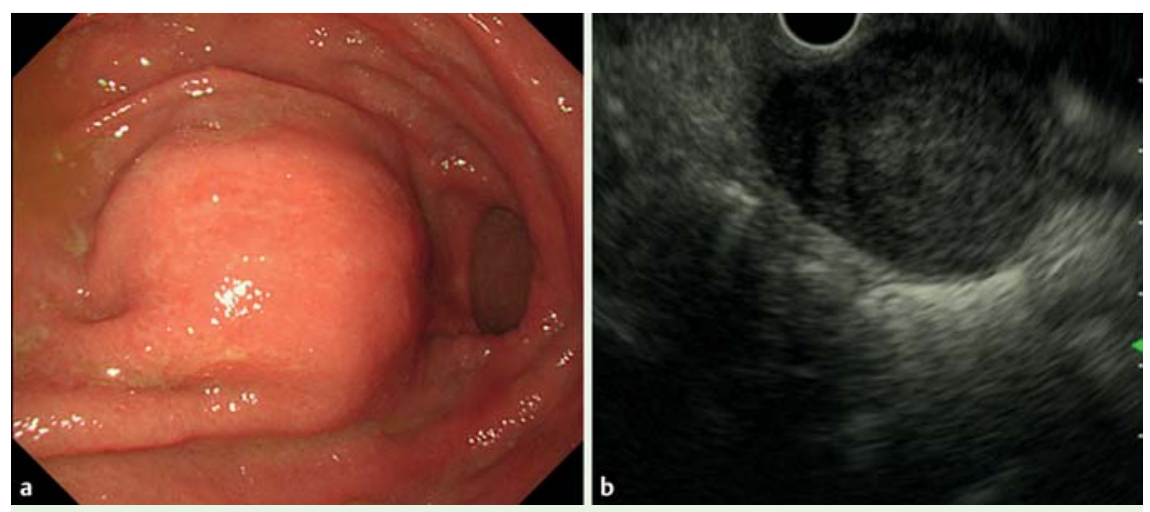

Fig. 2 The submucosal tumor in the greater curvature of the remnant stomach shown: a during esophagogastroduodenoscopy; b on endoscopic ultrasound (EUS), which showed the tumor to be in the fourth layer.

gastric carcinoma presented with a desmoid tumor of the remnant stomach, although this was situated in a different part of the stomach and well away from the resection line of the previous gastric surgery. Complete surgical resection with negative margin remains the only curative treatment option for desmoid tumor [4].

Endoscopy_UCTN_Code_CCL_1AB_2AD_3AB

\section{Competing interests: None}

Keiichi Date', Yasuo Shima',

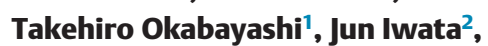
Tatsuaki Sumiyoshi ${ }^{1}$, Akihito Kozuki ${ }^{1}$

${ }^{1}$ Department of Gastroenterological Surgery, Kochi Health Sciences Center, Kochi, Japan

2 Department of Diagnostic Pathology, Kochi Health Sciences Center, Kochi, Japan 


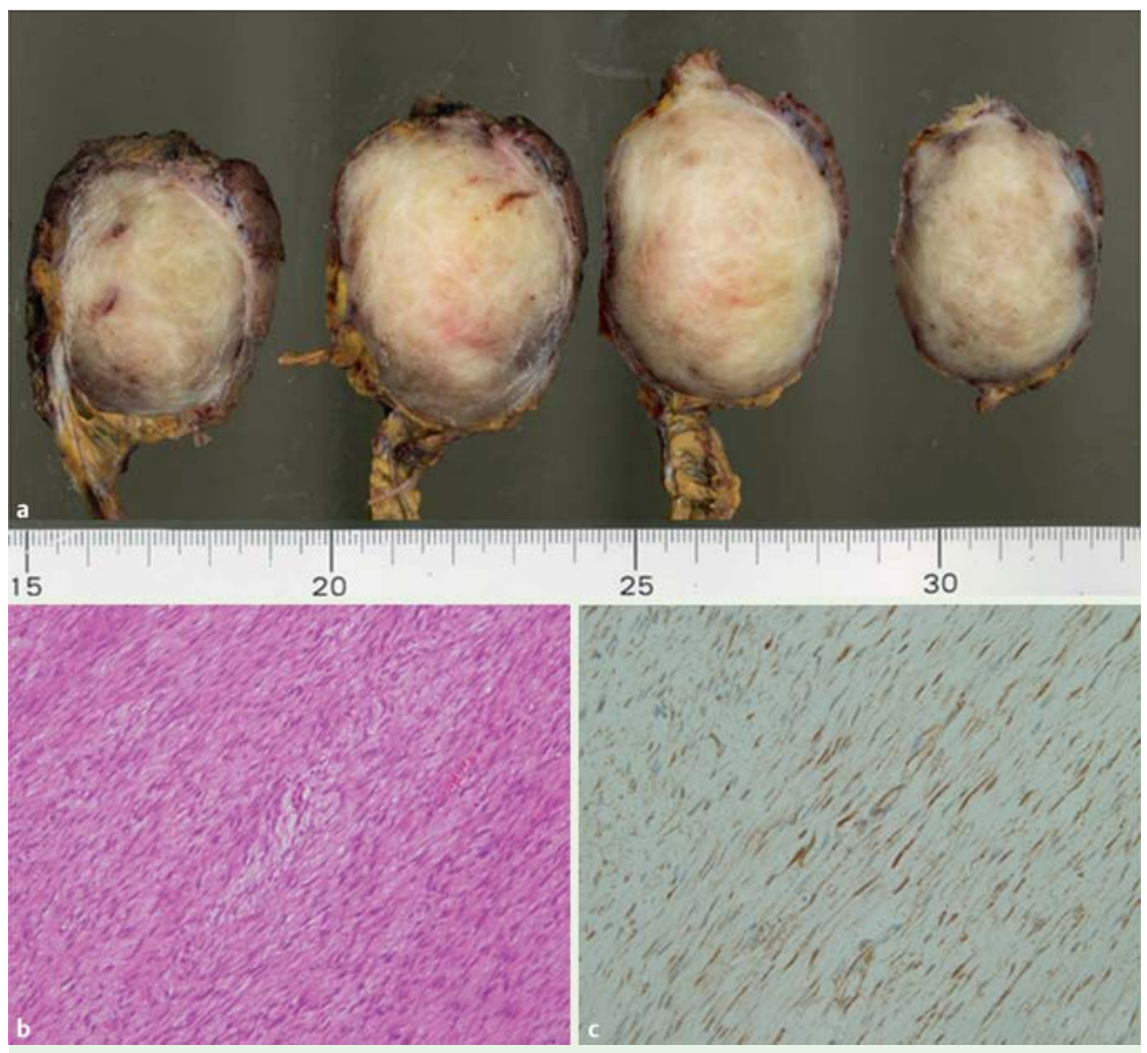

Fig. 3 Pathological examination of the resected specimen showed: a macroscopically, a tumor measuring $40 \times 40 \mathrm{~mm}$, with a whitish, uniform cut surface; $\boldsymbol{b}$ sheets of spindle-shaped cells with bland nuclear morphology and fine chromatin on hematoxylin and eosin (H\&E) staining (magnification $\times 20$ ); c tumor cells that were positive on immunostaining with beta-catenin (magnification $\times 40$ ).

\section{References}

1 Kabiri EH, Al Aziz S, El Maslout A et al. Desmoid tumors of the chest wall. Eur J Cardiothorac Surg 2001; 19: 580-583

2 Economou A, Pitta X, Andreadis E et al. Desmoid tumor of the abdominal wall: a case report. J Med Case Rep 2011; 5: 326

3 Bolke E, Krasniqi H, Lammering $G$ et al. Chest wall and intrathoracic desmoid tumors: surgical experience and review of the literature. Eur J Med Res 2009; 14: 240-243

4 Polistina F, Costantin G, D'Amore $E$ et al. Sporadic, nontrauma-related, desmoid tumor of the pancreas: a rare disease-case report and literature review. Case Rep Med 2010; 2010: 272760

\section{Bibliography}

DOI http://dx.doi.org/

10.1055/s-0034-1391870

Endoscopy 2015; 47: E242-E243

(c) Georg Thieme Verlag KG

Stuttgart · New York

ISSN 0013-726X

\section{Corresponding author}

\section{Takehiro Okabayashi, MD, PhD}

Department of Gastroenterological Surgery Kochi Health Sciences Center 2125-1 Ike, Kochi-City Kochi 781-8555

Japan

Fax: +81-88-8376766

takehiro_okabayashi@khsc.or.jp 